OPEN ACCESS

Edited by:

Masakazu Toi,

Kyoto University, Japan

Reviewed by:

Binghe $X u$,

Chinese Academy of Medical

Sciences and Peking Union Medical

College, China

Tomás Pascual Martinez,

University of North Carolina at Chapel

Hill, United States

*Correspondence:

Wenjun $Y_{i}$

yiwenjun@csu.edu.cn

Quchang Ouyang

oyqc1969@126.com

tThese authors have contributed equally to this work

Specialty section:

This article was submitted to

Women's Cancer,

a section of the journal

Frontiers in Oncology

Received: 23 January 2020 Accepted: 24 April 2020

Published: 25 May 2020

Citation:

Chen Q, Ouyang D, Anwar M, Xie N,

Wang S, Fan P, Qian L, Chen G,

Zhou E, Guo L, Gu X, Ding B, Yang $X$,

Liu L, Deng C, Xiao Z, Li J, Wang Y,

Zeng S, Hu J, Zhou W, Qiu B,

Wang Z, Weng J, Liu M, Li Y, Tang T,

Wang J, Zhang H, Dai B, Tang W,

Wu T, Xiao M, LiX, LiU H, Li L, Yi W

and Ouyang Q (2020) Effectiveness

and Safety of Pyrotinib, and

Association of Biomarker With

Progression-Free Survival in Patients With HER2-Positive Metastatic Breast

Cancer: A Real-World, Multicentre Analysis. Front. Oncol. 10:811. doi: 10.3389/fonc.2020.00811

\section{Effectiveness and Safety of Pyrotinib, and Association of Biomarker With Progression-Free Survival in Patients With HER2-Positive Metastatic Breast Cancer: A Real-World, Multicentre Analysis}

\author{
Qitong Chen ${ }^{1 \dagger}$, Dengjie Ouyang ${ }^{1,2+}$, Munawar Anwar ${ }^{1}$, Ning Xie ${ }^{3}$, Shouman Wang ${ }^{4}$, \\ Peizhi Fan ${ }^{5}$, Liyuan Qian ${ }^{6}$, Gannong Chen ${ }^{1}$, Enxiang Zhou ${ }^{1}$, Lei Guo ${ }^{4}$, Xiaowen Gu ${ }^{5}$, \\ Boni Ding ${ }^{6}$, Xiaohong Yang ${ }^{7}$, Liping Liu ${ }^{7}$, Chao Deng ${ }^{8}$, Zhi Xiao ${ }^{3}$, Jing $\mathrm{Li}^{9}$, Yunqi Wang ${ }^{10}$, \\ Shan Zeng ${ }^{11}$, Jinhui Hu ${ }^{12}$, Wei Zhou ${ }^{13}$, Bo Qiu ${ }^{14}$, Zhongming Wang ${ }^{15}$, Jie Weng ${ }^{16}$, \\ Mingwen Liu ${ }^{17}$, Yi Li ${ }^{18}$, Tiegang Tang ${ }^{19}$, Jianguo Wang ${ }^{20}$, Hui Zhang ${ }^{21}$, Bin Dai ${ }^{22}$,

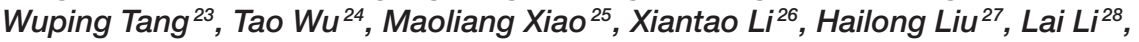 \\ Wenjun $\mathrm{Yi}^{1 *}$ and Quchang Ouyang ${ }^{3 *}$
}

${ }^{1}$ Department of General Surgery, The Second Xiangya Hospital, Central South University, Changsha, China, ${ }^{2}$ Department of General Surgery, Xiangya Hospital Central South University, Changsha, China, ${ }^{3}$ Department of Breast Internal Medicine, The Affiliated Cancer Hospital of Xiangya School of Medicine, Central South University, Changsha, China, ${ }^{4}$ Department of Breast Surgery, Xiangya Hospital, Central South University, Changsha, China, ${ }^{5}$ Department of Breast and Thyroid Surgery, Hunan Provincial People's Hospital, Changsha, China, ${ }^{6}$ Department of Breast and Thyroid Surgery, Central South University Third Xiangya Hospital, Changsha, China, ${ }^{7}$ Department of Oncology, The Affiliated Cancer Hospital of Xiangya School of Medicine, Cental South University, Changsha, China, ${ }^{8}$ Department of Oncology, The Second Xiangya Hospital, Central South University, Changsha, China, ${ }^{9}$ Department of Breast Medical Oncology, The Affiliated Cancer Hospital of Xiangya School of Medicine, Cental South University, Changsha, China, ${ }^{10}$ Department of Traditional Chinese Medicine, The Affiliated Cancer Hospital of Xiangya School of Medicine, Cental South University, Changsha, China, ${ }^{11}$ Department of Internal Medicine Oncology, Xiangya Hospital Central South University, Changsha, China, ${ }^{12}$ Department of Breast Surgery, The First Hospital Hunan University of Chinese Medicine, Changsha, China, ${ }^{13}$ Department of Breast and Thyroid Surgery, The Affiliated ZhuZhou Hospital of Xiangya School of Medicine Central South University, Zhuzhou, China, ${ }^{14}$ Department of Oncology, The Affiliated ZhuZhou Hospital of Xiangya School of Medicine Central South University, Zhuzhou, China, ${ }^{15}$ Department of Breast Surgery, The Third People's Hospital of Yongzhou, Yongzhou, China, ${ }^{16}$ Department of Oncology, The First People's Hospital of Yueyang, Yueyang, China, ${ }^{17}$ Department of Breast and Thyroid Surgery, The First People's Hospital of Xiangtan City, Xiangtan, China, ${ }^{18}$ Department of Oncology, The Third People's Hospital of Changde, Changde, China, ${ }^{19}$ Department of Oncology, Xiangtan Central Hospital, Xiangtan, China, ${ }^{20}$ Department of General Surgery, Xiangtan Central Hospital, Xiangtan, China, ${ }^{21}$ Department of Oncology, Central Hospital of Shaoyang, Shaoyang, China, ${ }^{22}$ Department of Breast and Thyroid Surgery, Central Hospital of Shaoyang, Shaoyang, China, ${ }^{23}$ Department of Breast Surgery, Shaoyang Hospital of Traditional Chinese Medicine, Shaoyang, China, ${ }^{24}$ Department of Oncology, The First People's Hospital of Changde, Changde, China, ${ }^{25}$ Department of Oncology, The Third Hospital of Hunan University of Chinese Medicine, Zhuzhou, China, ${ }^{26}$ Department of Oncology, The Central Hospital of Yiyang, Yiyang, China, ${ }^{27}$ Department of Internal Medicine - Oncology, The First People's Hospital of Chenzhou, Chenzhou, China, ${ }^{28}$ Department of Breast and Thyroid Surgery, The People's Hospital of Xiangtan County, Xiangtan, China

Background: Pyrotinib, an irreversible pan-ERBB inhibitor, has shown promising antitumour activity, and acceptable tolerability. This research was conducted to evaluate the actual use and effectiveness of pyrotinib in China, therefore, contributed to solve the problem of real-world data scarcity.

Methods: In this retrospective study, 168 patients who received pyrotinib treatment for HER2-positive metastatic breast cancer (MBC) in Hunan Province from June 
2018 to August 2019 were included. Progression-free survival (PFS), tumor mutation burden (TMB), and drug-related adverse events (AEs) after pyrotinib administration were analyzed.

Results: The median PFS (mPFS) time in the 168 participants was 8.07 months. The mPFS times in patients with pyrotinib in second-line therapy $(n=65)$ and third-or-higher-line therapy $(n=94)$ were 8.10 months and 7.60 months, respectively. Patients with brain metastases achieved 8.80 months mPFS time. In patients with pyrotinib in third-or-higher-line therapy, patients who had previously used lapatinib still got efficacy but showed a shorter mPFS time (6.43 months) than patients who had not (8.37 months). TMB was measured in 28 patients, $\mathrm{K}-\mathrm{M}$ curve $(P=0.0024)$ and Multivariate Cox analysis $(P=0.0176)$ showed a significant negative association between TMB and PFS. Diarrhea occurred in $98.2 \%$ of participants (in any grade) and $19.6 \%$ in grade 3-4 AEs.

Conclusion: Pyrotinib is highly beneficial to second-or-higher-line patients or HER2-positive MBC patients with brain metastases. Pyrotinib seems to be a feasible strategy both in combination of chemotherapeutic drugs or as a replacement of lapatinib if diseases progressed. TMB could be a potential predictor for evaluating pyrotinib's effectiveness in HER2-positive MBC.

Keywords: breast cancer, HER2, pyrotinib, tumor mutation burden, metastases

\section{INTRODUCTION}

Approximately $20-30 \%$ of patients with breast cancer demonstrate overexpression of human epidermal growth factor receptor 2 (HER2) (1). This type of breast cancer exhibits more-aggressive clinical behavior and poorer outcomes than those who do not overexpress HER2 (1). With the development of anti-HER2 therapies, such as trastuzumab (2), pertuzumab (3), lapatinib (4), neratinib (5), and ado-trastuzumab emtansine (TDM1) (6), the prognoses of patients with HER2-positive breast cancer have improved significantly. However, resistance and AEs are frequently observed during HER2-directed therapy, and are obstacles to the continuous administration of these agents (7). Therefore, it is crucial to improve anti-HER2 strategies for patients who are intolerant of standard therapies, as well as determine the mechanisms of resistance.

Pyrotinib is an orally administered tyrosine-kinase inhibitor (TKI) that has been approved in China for the treatment of

\footnotetext{
Abbreviations: AEs, Adverse events; BBB, Blood-brain barrier; CDK12, Cyclin Dependent Kinase 12; CI, Confidence intervals; ctDNA, Circulating tumor DNA; DNMT3A, DNA Methyltransferase 3 Alpha; EGFR, Epidermal growth factor receptor; ECOG, Eastern Cooperative Oncology Group; ERBB, Erb-B2 Receptor Tyrosine Kinase; ER, Estrogen receptor; RECIST, Evaluation criteria in solid tumors; FISH, Fluorescence in situ hybridization; HER2, Human epidermal growth factor receptor 2; IHC, Immunohistochemistry; MBC, Metastatic breast cancer; NGS, Next-generation sequencing; NOTCH4, Notch Receptor 4; PFS, Progression-free survival; PIK3CA, Phosphatidylinositol-4,5-Bisphosphate 3Kinase Catalytic Subunit Alpha; PR, Progesterone receptor; S-1, Tegafur, gimeracil and oteracil Potassium; SMARCA4, SWI/SNF related, matrix associated, Actin dependent regulator of Chromatin, subfamily A, member 4; MLL2, Lysine methyltransferase 2D; T-DM1, Ado-trastuzumab emtansine; TKI, Tyrosine-kinase inhibitor; TMB, Tumor mutation burden; TP53, Tumor protein P53; mPFS, Median progression-free survival.
}

HER2-positive MBC (8). Preclinical data suggest that pyrotinib can irreversibly inhibit multiple receptor tyrosine kinases of the ERBB family (including HER1 [also known as epidermal growth factor receptor (EGFR)], HER2, and HER4), and effectively inhibit the proliferation of HER2-overexpressing cells both in vivo and in vitro $(9,10)$. Efforts are being made to evaluate the in vivo efficacy and safety of pyrotinib, and to determine the associated AEs. In a phase I pyrotinib-monotherapy study and a phase II pyrotinib-vs.-lapatinib study, the recommended dosage of oral pyrotinib was $400 \mathrm{mg}$ once daily after a meal $(11,12)$. Whether monotherapy or combined therapy can lead to significantly improved objective response rates and PFS times with controllable toxicity (e.g., diarrhea) $(11,12)$. Although phase III clinical trials are in progressing, it cannot fully reflect the real-world treatment setting as there is lack of relevant data.

Besides real-world data to evaluate pyrotinib efficacy in the treatment of breast cancer, it is important to identify biomarkers to predict effectiveness of pyrotinib-based therapy. Although PIK3CA and TP53 were found to be associated with low treatment efficacy of pyrotinib monotherapy in phase I study (11), this correlation was not observed in pyrotinib in combination with capecitabine therapy (13). Thus, these contrary results suggest that better indicators need to be explored to evaluate the efficacy of pyrotinib-based therapy. Currently, TMB is emerging as an outcome biomarker of immune checkpoint blockade response (14). The implication of TMB in other treatment settings, such as targeted therapy, is little unknown. Studies have shown that TMB can be used as a therapeutic marker of EGFR-TKI for lung cancer (15-17). Nevertheless, there are lack of researches focus on investigating the relationship between TMB and treatment outcomes in HER2-positive MBC, especially for pyrotinib-based treatments. 
By analyzing real-world data from a multicentre study of patients with HER2-positive MBC who were treated with pyrotinib, this study aimed to evaluate the effects on PFS of the pyrotinib treatment line, the metastatic site, the use of pyrotinib in combination with other chemotherapeutic agents, and replacement of lapatinib. Simultaneously, the relationship between TMB and the outcome of pyrotinib treatment has been analyzed, in order to identify potential predictive or prognostic biomarkers for HER2-positive MBC. Finally, the AEs associated with pyrotinib treatment were also analyzed in this study.

\section{PATIENTS AND METHODS}

\section{Patient Eligibility and Study Design}

The study used the following inclusion criteria: (i) eligible patients had a confirmed histological or cytological diagnosis of HER2-positive MBC (with tumor tissue protein expression demonstrated by immunohistochemistry [IHC] category $3+$ or positive results of fluorescence in situ hybridization [FISH]); (ii) eligible patients had a measurable lesion as defined by the revised Response Evaluation Criteria in Solid Tumors guidelines version 1.1 (RECIST 1.1); (iii) eligible patients had adequate hematological, hepatic, and renal functions. No limits on the number of prior cytotoxic regimens for metastatic disease were set.

Patients were excluded if they discontinued pyrotinib treatment, either because of medication use in a neoadjuvant setting $(n=7)$, or for reasons unrelated to treatment progress [economic reasons $[n=27$ ], severe AEs $[n=18]$ ], or if they were lost to follow-up for other, unknown reasons $(n=12)$ (Supplementary Figure 1).

This study was a multicentre $(n=20)$, retrospective, real-world study (RWS) conducted from the Second Xiangya Hospital of Central South University (Hunan Province, China). Participants were women with $\mathrm{MBC}$ who started treatment with pyrotinib administered in standard clinical practice in one of the hospitals in Hunan Province. Patients received either $400 \mathrm{mg}$ pyrotinib $(n=153,91.1 \%)$ or $320 \mathrm{mg}$ pyrotinib $(n=15,8.9 \%)$ once daily, in 21-day cycles, in addition to other medication as indicated in Table 1. All patients provided their written informed consent, and the study was reviewed and approved by the Research Ethics Committee of the Second Xiangya Hospital of Central South University.

\section{Data Collection}

Data collection was performed retrospectively by two trained staff using a standardized data-collection method from the routine clinical information system, and were documented in an electronic case-report form. Data were monitored using automated plausibility checks and on-site monitoring.

First-line treatment was defined as treatment for a patient with de novo stage IV breast cancer not treated previously with anti-HER2 medications, or for a patient with recurrence $>12$ months after discontinuation of trastuzumab. Second-line treatment was that given to a patient with recurrence within 12 months of discontinuation of trastuzumab, or recurrence during adjuvant therapy with trastuzumab, or progression following
TABLE 1 | Characteristics of 168 patients with HER2-positive MBC

\begin{tabular}{|c|c|c|c|}
\hline & $\begin{array}{c}\text { Total } \\
(n=168)\end{array}$ & $\%$ & Median PFS (95\% Cl) \\
\hline Median age (range), years & $50(28-73)$ & & \\
\hline$<50$ & 82 & 48.81 & 7.73 (6.624-8.836) \\
\hline$\geq 50$ & 86 & 51.19 & $8.67(7.242-10.098)$ \\
\hline \multicolumn{4}{|l|}{ ECOG scale } \\
\hline $0-1$ & 155 & 92.26 & $8.17(7.149-9.191)$ \\
\hline$\geq 2$ & 13 & 77.38 & $7.03(5.917-8.143)$ \\
\hline \multicolumn{4}{|l|}{ Menopausal status } \\
\hline Postmenopausal & 93 & 55.36 & $7.47(6.723-8.217)$ \\
\hline Premenopausal & 75 & 44.64 & 8.97 (7.739-10.201) \\
\hline \multicolumn{4}{|l|}{ Hormone-receptor status } \\
\hline Positive & 90 & 53.57 & $8.00(6.468-9.532)$ \\
\hline Negative & 72 & 42.86 & $8.67(7.269-10.071)$ \\
\hline Unknown & 6 & 3.57 & $5.80(0.000-12.113)$ \\
\hline \multicolumn{4}{|l|}{ Treatment stage } \\
\hline First-line & 9 & 5.36 & - \\
\hline Second-line & 65 & 38.69 & $8.17(6.466-9.874)$ \\
\hline Third-or-higher-line & 94 & 55.95 & $7.60(6.352-8.848)$ \\
\hline \multicolumn{4}{|l|}{ Treatment type } \\
\hline \multicolumn{4}{|l|}{ No previous use of capecitabine } \\
\hline Pyrotinib + capecitabine & 114 & 67.86 & $8.67(7.110-10.230)$ \\
\hline \multicolumn{4}{|l|}{ Previous use of capecitabine } \\
\hline Pyrotinib + abraxane & 19 & 11.31 & $8.70(4.405-12.995)$ \\
\hline Pyrotinib + trastuzumab & 12 & 7.14 & $5.20(0.000-10.683)$ \\
\hline Pyroti nib + others & 23 & 13.69 & 6.67 (5.653-7.687) \\
\hline \multicolumn{4}{|l|}{ Metastatic site } \\
\hline Soft tissue and/or bone & 30 & 17.86 & $6.70(5.145-8.255)$ \\
\hline Organ & 99 & 58.93 & 8.07 (6.741-9.399) \\
\hline Brain & 39 & 23.21 & $8.80(6.588-11.012)$ \\
\hline
\end{tabular}

ECOG, Eastern Cooperative Oncology Group; PFS, progression-free survival; Cl, confidence interval.

first-line treatment. Third-or-higher-line treatment was that given to a patient with progression or recurrence following second-line treatment and for which any one of the anti-HER2 drugs or chemotherapeutic drugs had been changed.

AEs that were recorded considered at least possibly pyrotinibtreatment-related by the treating physician. The date of onset of AEs and their severity according to the National Cancer Institute Common Terminology Criteria for Adverse Events (version 4.0) were recorded.

\section{Definition of HER2 Status and Grading}

Information about the HER2 status, and grading were obtained for documentation purposes for each tumor that had been biopsied. Samples for these analyses originated from primary tumors, local recurrences, and metastatic sites. The biomarker status was determined for HER2 as follows: if a biomarker assessment of the metastatic site was available, this receptor status was used. If no information was available for metastases, the latest biomarker results from the primary tumor were used. All patients who had ever received anti-HER2 therapy were assumed to be 
HER2 positive. A positive HER2 status required an IHC score of $3+$ or positive FISH results according to the ASCO/CAP 2018 HER2 test guideline (18).

\section{Assessment of TMB}

In our cohort, 28 out of 168 peripheral-blood samples collected (only 28 patients consent) prior to pyrotinib treatment were subjected to a next-generation sequencing (NGS) assay (OncoMD/OncoMD Plus [Beijing, China], comprising a customized panel of 1,021 genes) at Geneplus-Beijing Institute (http://www.geneplus.org.cn), Beijing, China. The TMB was determined by integrated mutation profiling testing of actionable cancer targets within the same gene panel. TMB [mutations per megabase [mut/Mb]] was calculated from sequenced DNA (19).

\section{End Point and Assessments}

The primary end point was PFS, which was defined as the time from drug administration to death or disease progression (whichever came first), as assessed by the investigator, according to RECIST 1.1 criteria. The end point was analyzed in the full population of all 168 patients.

Six patients were censored at the last valid disease-assessment date or at the last prior assessment after two missed visits. Clinical follow-up was scheduled every 2-3 weeks during treatment. Imaging follow-up was scheduled every 1-2 months, according to the standard clinical practice.

\section{Statistical Analyses}

Pearson's $\chi^{2}$ test or Fisher's exact test were used for comparisons of categorical variables in different groups of patients. Continuous distributions which did not follow a normal distribution were compared using a non-parametric MannWhitney $U$-test. Kaplan-Meier estimates of PFS were compared between treatment arms using a log-rank test. Median survival times and 95\% confidence intervals (CI) were estimated. Univariable and multivariable Cox proportional hazard models were used for assessment of the adjusted effects of covariates on PFS. Statistical analyses were performed using SPSS statistical software version 22.0 (SPSS, NY, USA: IBM Corporation), $\mathrm{R}$ 3.6.3 and Prism analysis and graphing software version 8.0.1 (GraphPad, San Diego, USA). A two-sided $P<0.05$ was considered statistically significant.

\section{RESULTS}

\section{Baseline Characteristics and Patients' Outcomes}

Between June 2018 and August 2019, we enrolled 168 patients with HER2-positive MBC. Among these patients, the median age was 50 years (range 28-73 years). The specific baseline characteristics, mPFS times, and 95\% CIs associated with particular ranges are presented in Table $\mathbf{1 .}$
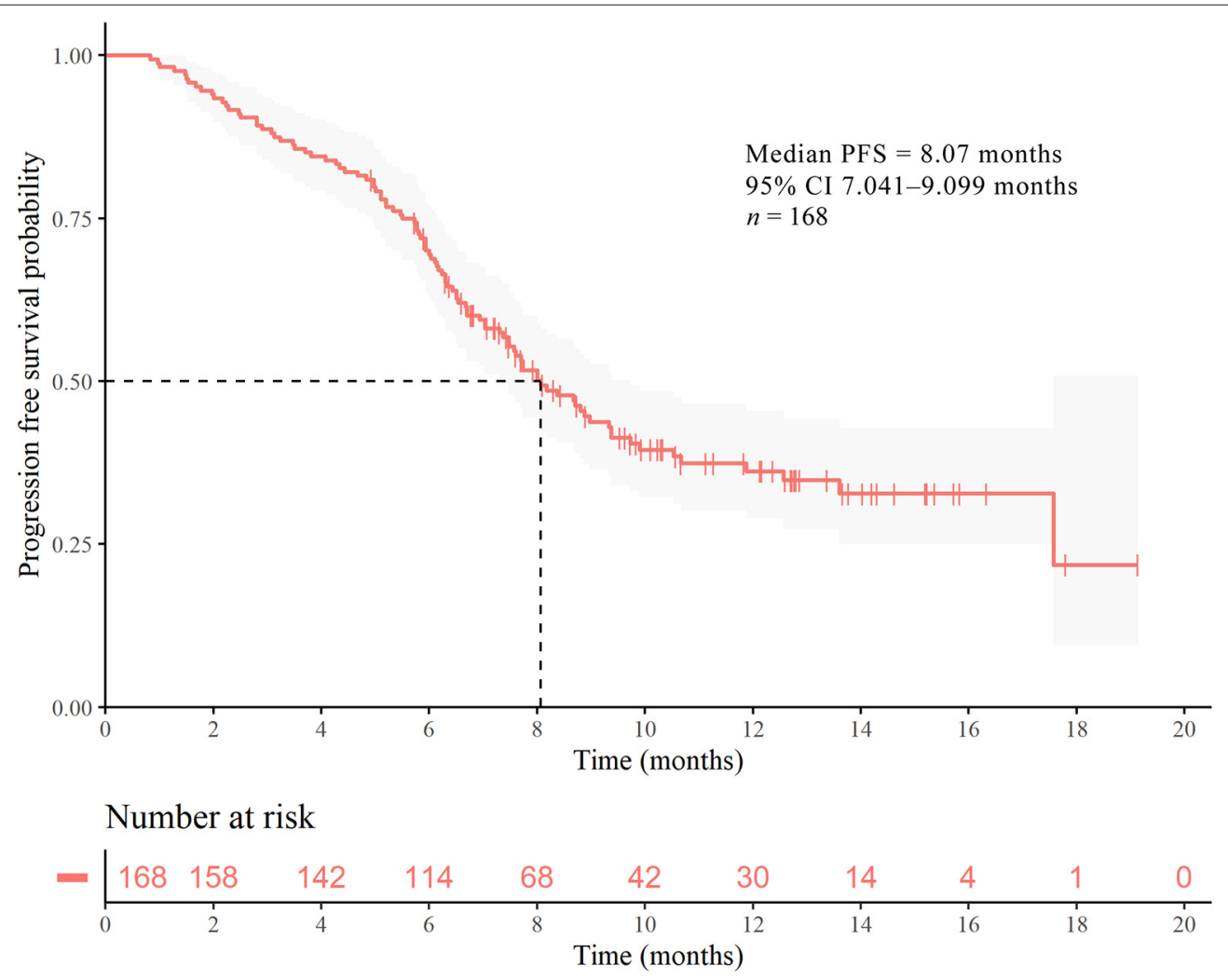

FIGURE 1 | Kaplan-Meier curves of PFS for patients with HER2-positive MBC. Survival analysis for the entire cohort $(n=168)$. 
Following up to January 2020, the median follow-up time was 7.30 months. The mPFS time in the study population was 8.07 months (95\% CI 7.041-9.099 months) (Figure 1). The number of PFS events was 99 (58.9\%). The mPFS time in patients with second-line pyrotinib treatment was 8.17 months, and the PFS for third-or-higher-line treatment was 7.60 months. There were too few progression events in the group of nine patients with first-line treatment to enable calculation of the mPFS. The mPFS time was shorter for third-or-higher-line pyrotinib treatment than for first-line and second-line treatment $(P=0.3266$, the difference is not significant, Figure 2A). Eighteen patients (10.71\%) achieved complete response (CR) and 50 (29.76\%) achieved partial response (PR) for an objective response rate (ORR) of $40.47 \%$ (95\% CI, 22.7-54.2\%).
Log-rank test indicated that age $(P=0.9137)$, hormonereceptor status $(P=0.7251)$, and classification of metastatic sites $(P=0.5828$, Figure 2B) at the time of pyrotinib initiation had no significant associations with PFS. The mPFS time in patients ( $n=39,23.21 \%$ ) with brain metastases, including those who also had metastases to other organs and those with local metastases, was 8.80 months. the mPFS for patients $(n=99,58.93 \%)$ with organs metastases was 8.07 months and patients $(n=30,17.86 \%)$ with soft tissue and/or bone metastases was 6.70 months. In patients treated with the combination of pyrotinib (pyrotinib with capecitabine, abraxane, trastuzumab, vinorelbine, etoposide, or S-1), our results suggested a significant difference PFS times ( $P$ $=0.0428$, Figure 2C). The PFS time of the pyrotinib combined with capecitabine group was 8.67 months while referring to patients who previously used capecitabine, pyrotinib combined
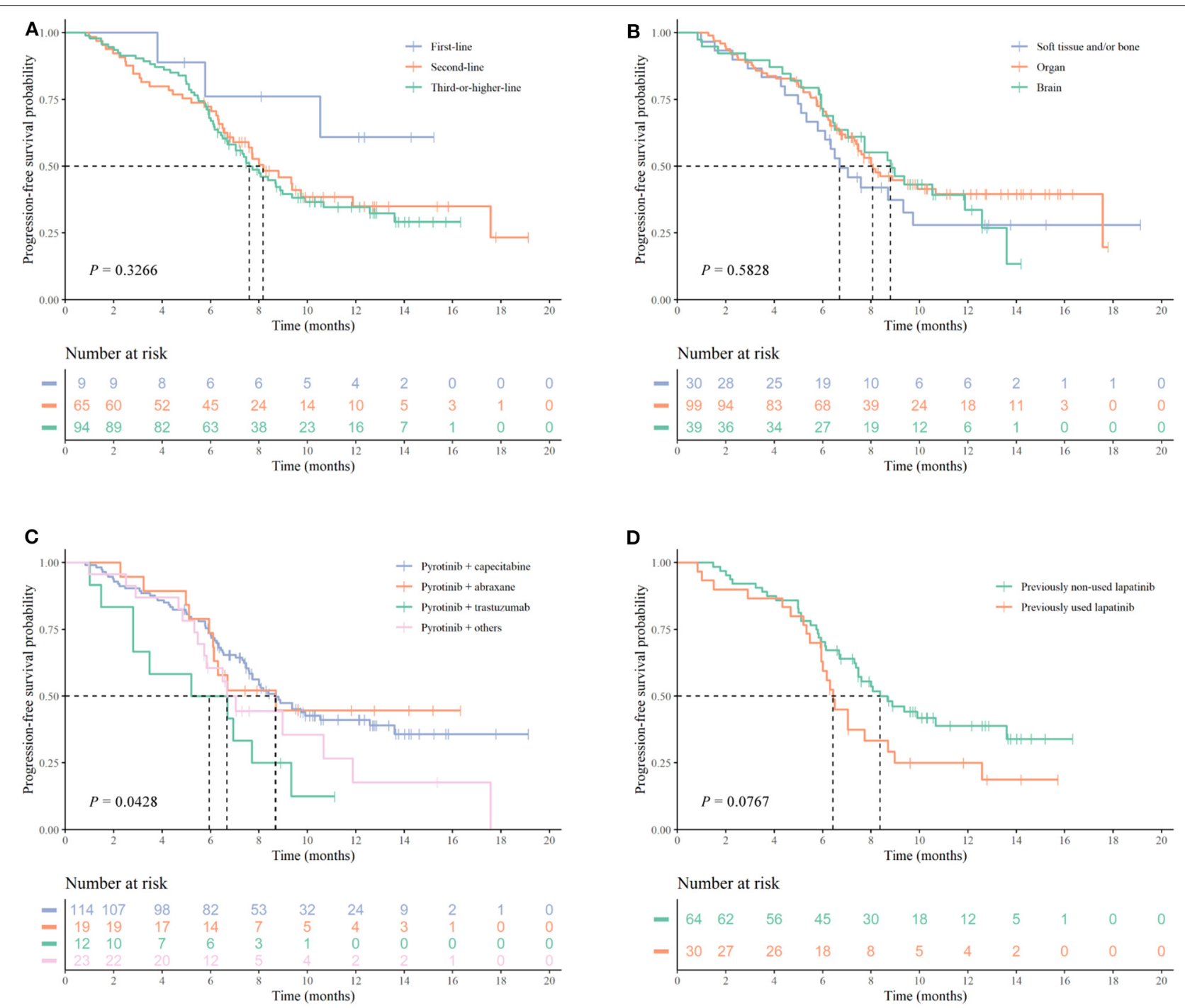

FIGURE 2 | Kaplan-Meier curves of PFS for patients with HER2-positive MBC. (A) Survival analysis comparing first-line $(n=9)$, second-line $(n=66)$, and third-or-higher-line ( $n=93$ ) pyrotinib-containing treatments. (B) Survival analysis according to metastatic sites. (C) Survival analysis according to treatment regimens. (D) Survival analysis according to previously used lapatinib $(n=30)$ or not $(n=64)$. P-values are from univariate log-rank tests. 
with abraxane was 8.70 months, pyrotinib with trastuzumab was 5.20 months, and pyrotinib combined with other drugs was 6.67 months.

\section{Effectiveness of Switching Use of TKIs}

In patients with anti-her2 treatment of third-or-higher-line treatment, patients who had previously used lapatinib $(n=$

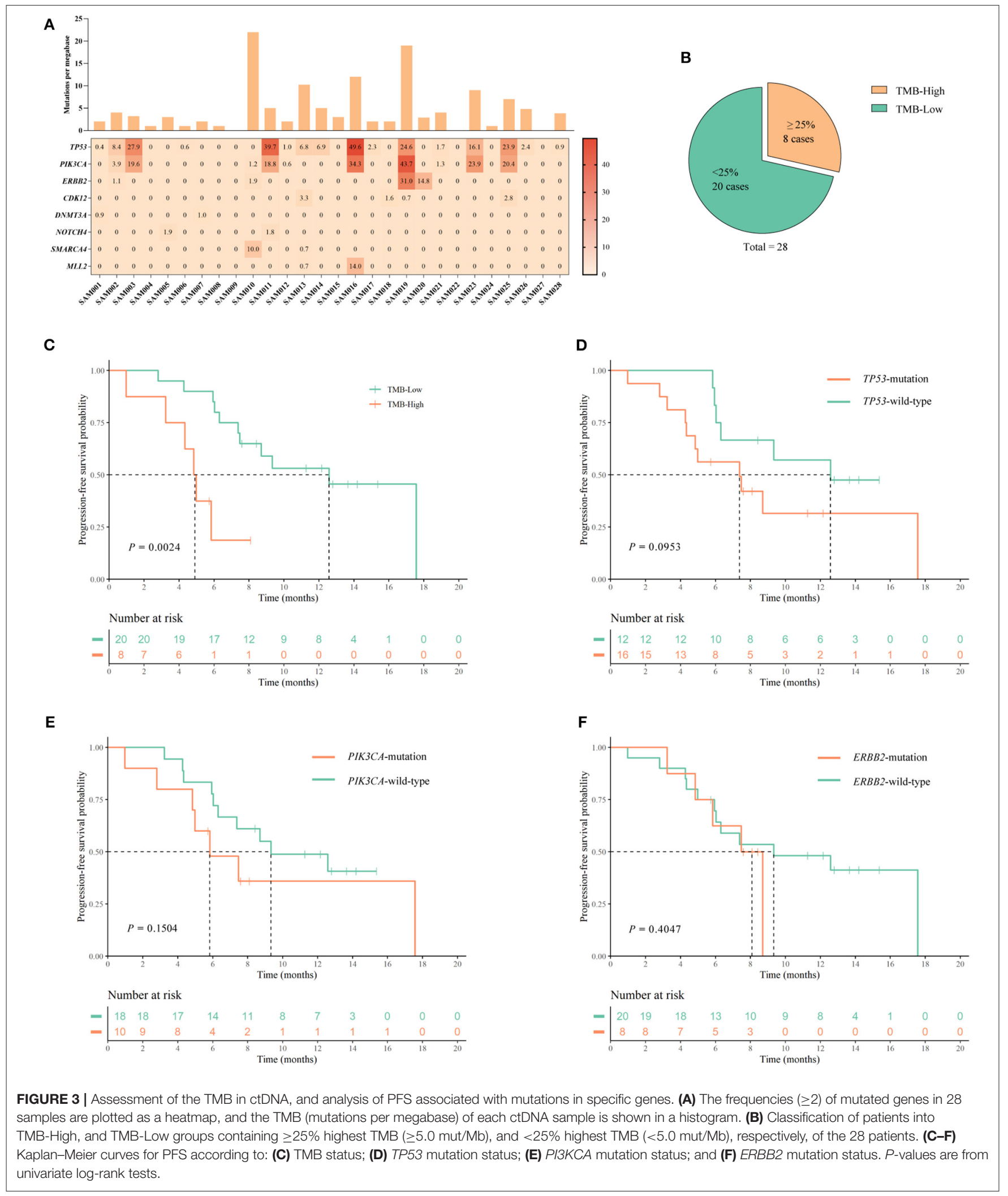


30 mPFS: 6.43 months 95\% CI 5.883-6.977 months) showed a different $(P=0.0767$, Figure 2D) PFS than patients who had not used lapatinib ( $n=64$ mPFS: 8.37 months 95\% CI 6.402-10.338 months). There was no statistical difference in the baseline data between the two groups (Supplementary Table 1).

\section{High TMB Is Associated With Poor PFS}

Next, we assessed the potential of tumor mutations detected in ctDNA as predictive biomarkers of pyrotinib effectiveness. Blood samples were available from 28 out of 168 patients, and NGS was performed on ctDNA. Detection of ctDNA mutations is summarized in Figures 3A,B. Among 28 patients, 21 (75.0\%) had a ctDNA TMB of $>1$ mut/Mb, and the median TMB per patient was $3 \mathrm{mut} / \mathrm{Mb}$ (range $0-22 \mathrm{mut} / \mathrm{Mb}$, mean $=4.71 \mathrm{mut} / \mathrm{Mb}$ ). The ctDNA TMB was $>3$ mut/Mb in 13 patients $(46.4 \%)$, and $\geq 6$ mut/Mb in six patients (21.4\%).

The 28 patients were assigned to the following TMB categories: the eight patients $(\geq 25 \%, \geq 5.0 \mathrm{mut} / \mathrm{Mb})$ with the highest TMB were classified as high TMB (TMB-H), and 20 patients $(<25 \%,<5.0$ mut $/ \mathrm{Mb})$ were low TMB (TMB-L) like other studies (20) (Figure 3B). There were no statistical differences in baseline characteristics between the high and low TMB groups (Supplementary Table 2). We compared the Kaplan-Meier PFS curves associated with these two TMB categories, and found a significant difference according to the log-rank test $(P=0.0024)$ (Figure 3C). mPFS values were 12.57 months (95\% CI 8.998-16.142) for TMB-L and 4.83 months (95\% CI 3.943-5.717 months) for TMB-H. These data suggest that high TMB may be a prognostic marker for poor PFS in patients undergoing HER2-directed treatments and chemotherapy. In comparisons of Kaplan-Meier PFS curves associated with the presence or absence of mutations in the individual TP53 $(n=$ $16)$, PIK3CA $(n=10)$, and ERBB2 $(n=8)$ genes, no significant differences were identified by log-rank tests (Figures 3D-F).

Univariate Cox analysis revealed that only TMB categorization $(P=0.0054)$ was significantly associated with PFS. Multivariable Cox regression analyses showed that metastatic site $(P=0.0498)$, treatment stage $(P=0.0343)$, and
TMB status $(P=0.0176)$ might be associated with PFS. Results of univariate and multivariate Cox analysis were presented in Table 2.

\section{AEs}

Diarrhea was the most common AE observed in our cohort, occurring in $98.7 \%$ of participants. Diarrhea was mainly grade $1-2$, but in $19.6 \%$ of patients was grade 3-4 (Table 3). Apart from diarrhea, the most frequent grade 3-4 AEs were nausea and vomiting (in $7.7 \%$ of patients), leukopenia (in $7.7 \%$ of patients), and hand-foot syndrome (in $6.5 \%$ of patients) (Table 3 ).

\section{Discussion}

In this study, pyrotinib was orally administered to patients with HER2-positive MBC. The study reached its primary end point. Generally, the result of data analysis demonstrates that pyrotinib treatment led to a median survival time of 8.07 months. This realworld research is a crucial complement to current clinical trials of pyrotinib.

Currently, the international recommended treatment regimen for HER2-positive MBC is trastuzumab plus pertuzumab with docetaxel; the second choice is TDM1, lapatinib or pertuzumab plus chemotherapy (21). Due to the diverse limitations of drug use in China, the preferred treatment is trastuzumab with docetaxel, and the fall-back plans include pyrotinib or lapatinib combined with capecitabine or trastuzumab overline therapy (21). Evidence suggests that in the second-line treatment regimen, the strategy of trastuzumab plus pertuzumab combined with capecitabine chemotherapy maximizes PFS to 11.1 months (22). By comparison, T-DM1 (6) and lapatinib in combination with capecitabine (4), have demonstrated PFS of 9.6 and 8.4 months, respectively. In this study, the PFS for patients with HER2-positive MBC was observed to be 8.17 months and 7.60 months for second-line and third-or-higher-line pyrotinib treatments separately. Pyrotinib acts directly on the intracellular tyrosine-kinase domain, which is unlike traditional anti-HER2 therapy, and can completely block the downstream pathway activation that results from ligand binding to ERBB-family

TABLE 2 | Univariable and multivariable analysis of factors for prediction of PFS in 28 patients with HER2-positive metastatic breast cancer who had NGS analysis.

\begin{tabular}{|c|c|c|c|c|}
\hline \multirow[t]{2}{*}{ Characteristics } & \multicolumn{2}{|c|}{ Univariable cox } & \multicolumn{2}{|c|}{ Multivariable cox } \\
\hline & HR (95\% Cl) & $P$-value & HR (95\% Cl) & $P$-value \\
\hline Age & 0.9736 (0.3499-2.7089) & 0.9592 & 0.1299 (0.0085-1.9665) & 0.1409 \\
\hline Menopausal_status & $1.3921(0.5132-3.7760)$ & 0.5158 & $3.2431(0.2570-40.921)$ & 0.3630 \\
\hline Hormone-receptor status & $0.5628(0.2249-1.4085)$ & 0.2194 & 0.6746 (0.1688-2.6959) & 0.5776 \\
\hline Metastatic site & 0.6648 (0.3187-1.3869) & 0.2766 & $0.3254(0.1059-0.9993)$ & 0.0498 \\
\hline Drug line & $1.3421(0.5342-3.3715)$ & 0.5312 & 3.8687 (1.1048-13.546) & 0.0343 \\
\hline Durg combination & $1.2336(0.8342-1.8241)$ & 0.2927 & $1.3745(0.7464-2.5312)$ & 0.3070 \\
\hline $\mathrm{TMB}$ & $5.2778(1.6332-17.055)$ & 0.0054 & $13.547(1.5752-116.50)$ & 0.0176 \\
\hline TP53 & 2.1558 (0.7610-6.1067) & 0.1481 & 1.0838 (0.2226-5.2767) & 0.9206 \\
\hline PIKЗCA & $1.4426(0.4782-4.3514)$ & 0.5153 & $0.9786(0.2461-3.8900)$ & 0.9755 \\
\hline ERBB2 & $1.7936(0.6352-5.0642)$ & 0.2699 & 1.1438 (0.1457-8.9753) & 0.8982 \\
\hline
\end{tabular}

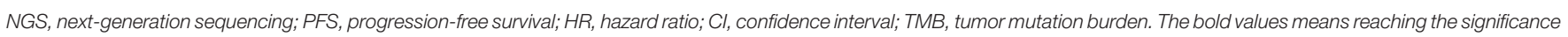
of statistics. 
TABLE 3 | Pyrotinib-related AEs of all grades and grade 3-4.

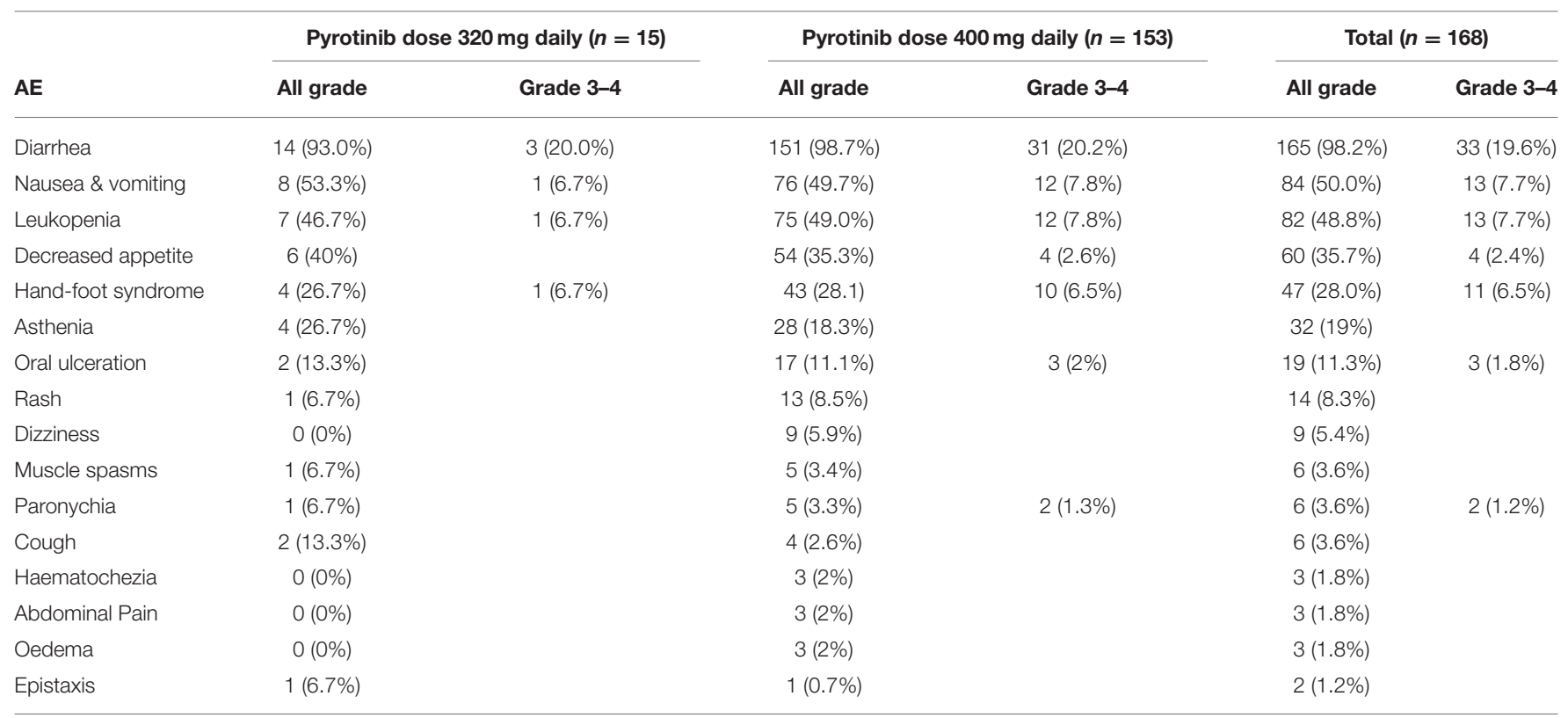

Pyrotinib-related adverse events (AES) includes definitely related AEs and probably related AES.

homodimers and heterodimers (10). This irreversible binding mode enhances the effects of pyrotinib and it also suggests drug resistance associated with HER2 over-expression may be overcame by pyrotinib (23). The results perform that pyrotinib could offer a breakthrough treatment in a second-or-higher-line treatment setting to some extent.

Brain metastases frequently occur in breast cancer, especially in HER2-positive MBC, for which the rate of brain metastasis is reportedly as high as $20-50 \%$ (24). Continuous anti-HER2 treatment after brain metastasis in HER2-positive breast cancer can reduce the risk of death from extracranial metastases by $\sim 50 \%$ (25). Trastuzumab, as a macromolecular monoclonal antibody, is not easy to cross the blood-brain barrier (BBB) (26). TKIs such as lapatinib performed a higher rate on crossing the $\mathrm{BBB}$ to enter the brain than trastuzumab, together with higher concentration surrounded in the brain metastases. (27). In clinical trials, lapatinib (28) or neratinib plus capecitabine (29) have resulted in PFS of 4.6 and 5.5 months in patients with brain metastases. T-DM1 has led to PFS of 5.9 months (30). In the present study, we found PFS of 8.80 months with pyrotinib in patients with brain metastases. Consequently, as a novel small molecule TKI, pyrotinib is significantly to improve the prognosis of patients with brain metastasis, although it requires further study for investigating the mechanisms of entering $\mathrm{BBB}$ and effectiveness of patients with brain metastasis.

Results from PHENIX study showed that the PFS time of pyrotinib combined with capecitabine could reach 11.1 months, together with an optimal PFS of 6.9 months for patients with brain metastasis (31). The differences existed between PHENIX study and this study might be caused by different population sample size and cohort. In PHENIX study, 79.5\% patients were visceral metastasis; patients with brain metastases or with thirdor-higher treatment lines accounted for 11.4 and $8.8 \%$ separately (31), while these patients accounted for a higher proportion in present study. Furthermore, the effectiveness of pyrotinib combined with other chemotherapeutic drugs in patients who had previously used capecitabine was compared with pyrotinib plus capecitabine in this study. The result indicated that the treatment strategy of pyrotinib plus abraxane has no statistical difference from pyrotinib plus capecitabine, while it is superior to the combination of pyrotinib plus trastuzumab or other chemotherapy. In other words, if disease progressed in those HER2-positive MBC patients treated by capecitabine, it is possible to choose other chemotherapy drugs to combine with pyrotinib.

There is a lack of research on switching utilization of TKIs. PHENIX study excluded patients who were treated with lapatinib (31). Present study explored the switching use of TKIs antiHER2 therapy, the result illustrates that taking pyrotinib after the initial use of lapatinib in third-or-higher treatment lines still got efficacy. Hence, it suggests that TKIs replacement could also be beneficial to patients while further investigations are still required.

Previous studies of the predictive values of tumor mutations have been based on analyses of primary tumor samples (32, 33). However, because of intratumour heterogeneity of gene profiles, biopsies of the primary tumor cannot reflect the whole picture of gene mutation in the patient, especially in individuals with different mutation status in the primary and metastatic tumors $(34,35)$. ctDNA has been suggested as an alternative to tumor biopsy samples for mutational analysis (36). Genetic analysis in HER2-positive MBC should take account of the multiple signal-transduction pathways [especially the activation 
of PI3K/Akt/mTOR pathway $(37,38)$ and ERBB2 mutation (23)] that are associated with resistance to anti-HER2 therapy. In addition to individual mutations, this study evaluated the overall $\mathrm{TMB}$ as a predictor of pyrotinib effectiveness. Previous study showed that high TMB has been identified as a prognostic marker for good overall survival in patients with HER2-positive MBC who undergo conventional HER2-directed treatments and chemotherapy (39). In the current study, high TMB was associated with poor PFS under pyrotinib-based treatment. This conversely relationship between TMB and PFS might be related to sample and antibody-dependent cells mediate cytotoxic effects of trastuzumab and pertuzumab. To the best of our knowledge, our study is the first to determine the association between ctDNA TMB and PFS in patients receiving pyrotinib therapy.

The incidence of pyrotinib-related AEs that we observed, such as diarrhea, nausea, vomiting et al. were similar with previous studies $(12,13,31)$. Diarrhea is the most common AE observed in present study. All pyrotinib-related AEs were effectively controlled with treatment and did not lead to discontinuation of pyrotinib treatment during the study. Notably, leukopenia was present in $49 \%$ of patients who received $400 \mathrm{mg}$ pyrotinib doses in our study, which was a higher incidence than that in a previous phase II study (46.2\%), probably because $32.1 \%$ of the patients included in our study were being treated in combination with other chemotherapeutic drugs.

The limitations of this study are the non-selectivity of the sample cohort in RWS and fewer patients in this study were previously treated with T-DM1 and pertuzumab. Therefore, the results of some studies are required for further verified by clinical randomized controlled studies.

In conclusion, this RWS contributed to validate the efficacy and safety of pyrotinib in advanced HER2-positive breast cancer. It brings benefits for second-or-higher-line patients, as well as provide more possible strategies for those with brain metastases. In addition to the current regimen of pyrotinib combined with capecitabine, pyrotinib can also be combined with other chemotherapeutic drugs, and the TKIs switching therapy is also beneficial to targeted-group patients. Furthermore, in this study, $\mathrm{TMB}$ is identified to be a possible candidate biomarker for

\section{REFERENCES}

1. Eroglu Z, Tagawa T, Somlo G. Human epidermal growth factor receptor family-targeted therapies in the treatment of HER2-overexpressing breast cancer. Oncologist. (2014) 19:135-50. doi: 10.1634/theoncologist.20 13-0283

2. Dawood S, Broglio K, Buzdar AU, Hortobagyi GN, Giordano SH. Prognosis of women with metastatic breast cancer by HER2 status and trastuzumab treatment: an institutional-based review. J Clin Oncol. (2010) 28:92-8. doi: 10.1200/JCO.2008.19.9844

3. Baselga J, Cortes J, Kim SB, Im SA, Hegg R, Im YH, et al. Pertuzumab plus trastuzumab plus docetaxel for metastatic breast cancer. New England J Med. (2012) 366:109-19. doi: 10.1056/NEJMoa1113216

4. Geyer CE, Forster J, Lindquist D, Chan S, Romieu CG, Pienkowski T, et al. Lapatinib plus capecitabine for HER2-positive advanced breast cancer. New England J Med. (2006) 355:2733-43. doi: 10.1056/NEJMoa064320 prediction of the response to pyrotinib-based therapy, which is desired to be further investigated in next steps.

\section{DATA AVAILABILITY STATEMENT}

The datasets generated for this study are available on request to the corresponding author.

\section{ETHICS STATEMENT}

All patients provided their written informed consent, and the study was reviewed and approved by the Research Ethics Committee of the Second Xiangya Hospital.

\section{AUTHOR CONTRIBUTIONS}

MA and QC collected and compiled the patients' information. QC and DO performed the literature search, data extraction and statistical analysis, and drafted the manuscript. WY and QO designed and supervised the study. Other authors conducted the clinical therapy of patients, followed up with the patients' information, and assess and recorded the AEs. All authors have read and approved the final manuscript.

\section{ACKNOWLEDGMENTS}

We thank all the hospitals and doctors who provided information of patients (name lists in Table Supplementary co-worker list).

\section{SUPPLEMENTARY MATERIAL}

The Supplementary Material for this article can be found online at: https://www.frontiersin.org/articles/10.3389/fonc. 2020.00811/full\#supplementary-material

Supplementary Figure 1 | Flow chart of the selection process for patients with HER-2-positive MBC who underwent pyrotinib treatment.

Supplementary Table 1 | The characteristic information of patients in pyrotinib third-or-higher-line therapy who have previously used lapatinib or not.

Supplementary Table 2 | Characteristics of 28 patients with HER2-positive metastatic breast cancer, according to TMB categorisation.

5. Burstein HJ, Sun Y, Dirix LY, Jiang Z, Paridaens R, Tan AR, et al. Neratinib, an irreversible ErbB receptor tyrosine kinase inhibitor, in patients with advanced ErbB2-positive breast cancer. J Clin Oncol. (2010) 28:1301-7. doi: 10.1200/JCO.2009.25.8707

6. Verma S, Miles D, Gianni L, Krop IE, Welslau M, Baselga J, et al. Trastuzumab emtansine for HER2-positive advanced breast cancer. New England J Med. (2012) 367:1783-91. doi: 10.1056/NEJMoa1209124

7. Loibl S, Gianni L. HER2-positive breast cancer. Lancet. (2017) 389:2415-29. doi: 10.1016/S0140-6736(16)32417-5

8. Blair HA. Pyrotinib: first global approval. Drugs. (2018) 78:1751-5. doi: 10.1007/s40265-018-0997-0

9. Zhu Y, Li L, Zhang G, Wan H, Yang C, Diao X, et al. Metabolic characterization of pyrotinib in humans by ultra-performance liquid chromatography/quadrupole time-of-flight mass spectrometry. $J$ Chromatogr B Analyt Technol Biomed Life Sci. (2016) 1034:117-27. doi: 10.1016/j.jchromb.2016.08.009 
10. Li X, Yang C, Wan H, Zhang G, Feng J, Zhang L, et al. Discovery and development of pyrotinib: a novel irreversible EGFR/HER2 dual tyrosine kinase inhibitor with favorable safety profiles for the treatment of breast cancer. Eur J Pharm Sci. (2017) 110:51-61. doi: 10.1016/j.ejps.2017.01.021

11. Ma F, Li Q, Chen S, Zhu W, Fan Y, Wang J, et al. Phase I study and biomarker analysis of pyrotinib, a novel irreversible Pan-ErbB receptor tyrosine kinase inhibitor, in patients with human epidermal growth factor receptor 2-positive metastatic breast cancer. J Clin Oncol. (2017) 35:3105-12. doi: 10.1200/JCO.2016.69.6179

12. Ma F, Ouyang Q, Li W, Jiang Z, Tong Z, Liu Y, et al. Pyrotinib or lapatinib combined with capecitabine in HER2-positive metastatic breast cancer with prior taxanes, anthracyclines, and/or trastuzumab: a randomized, phase II study. J Clin Oncol. (2019) 37:2610-9. doi: 10.1200/JCO.19.00108

13. Li Q, Guan X, Chen S, Yi Z, Lan B, Xing P, et al. Safety, efficacy, and biomarker analysis of pyrotinib in combination with capecitabine in HER2positive metastatic breast cancer patients: a phase i clinical trial. Clin Cancer Res. (2019) 25:5212-20. doi: 10.1158/1078-0432.CCR-18-4173

14. Thomas A, Routh ED, Pullikuth A, Jin G, Su J, Chou JW, et al. Tumor mutational burden is a determinant of immune-mediated survival in breast cancer. Oncoimmunology. (2018) 7:e1490854. doi: 10.1080/2162402X.2018.1490854

15. Offin M, Rizvi H, Tenet M, Ni A, Sanchez-Vega F, Li BT, Drilon A, et al. Tumor Mutation Burden and Efficacy of EGFR-Tyrosine Kinase Inhibitors in Patients with EGFR-Mutant Lung Cancers. Clin Cancer Res. (2019) 25:1063-9. doi: 10.1158/1078-0432.CCR-18-1102

16. Blakely CM, Watkins BKT, Wu W, Gini B, Chabon JJ, McCoach CE, et al. Evolution and clinical impact of co-occurring genetic alterations in advanced-stage EGFR-mutant lung cancers. Nat Genet. (2017) 49:1693-704. doi: 10.1038/ng.3990

17. Cheng ML, Oxnard GR. Does TMB impact the effectiveness of TKIs in EGFR-mutant NSCLC? Clin Cancer Res. (2019) 25:899-900. doi: 10.1158/1078-0432.CCR-18-2368

18. Wolff AC, Hammond EHM, Allison KH, Harvey BE, Mangu PB, Bartlett MST, et al. human epidermal growth factor receptor 2 testing in breast cancer: american society of clinical oncology/college of american pathologists clinical practice guideline focused update. Arch. Pathol. Lab. Med. (2018) 142:1364-82. doi: 10.5858/arpa.2018-0902-SA

19. Yang X, Chu Y, Zhang R, Han Y, Zhang L, Fu Y, et al. Technical validation of a next-generation sequencing assay for detecting clinically relevant levels of breast cancer-related single-nucleotide variants and copy number variants using simulated cell-free DNA. J Mol Diagn. (2017) 19:525-36. doi: 10.1016/j.jmoldx.2017.04.007

20. Zhang L, Liu Z, Li J, Huang T, Wang Y, Chang L, Zheng W, et al. Genomic analysis of primary and recurrent gliomas reveals clinical outcome related molecular features. Sci Rep. (2019) 9:16058. doi: 10.1038/s41598-019-52515-9

21. National Comprehensive Cancer Network, Breast cancer (version 2.2019).

22. Urruticoechea A, Rizwanullah M, Im SA, Ruiz CSA, Lang I, Tomasello G, et al. Randomized phase III trial of trastuzumab plus capecitabine with or without pertuzumab in patients with human epidermal growth factor receptor 2-positive metastatic breast cancer who experienced disease progression during or after trastuzumab-based therapy. J Clin Oncol. (2017) 35:3030-8. doi: 10.1200/JCO.2016.70.6267

23. Marty M, Cognetti F, Maraninchi D, Snyder R, Mauriac L, TubianaHulin $M$, et al. Randomized phase II trial of the efficacy and safety of trastuzumab combined with docetaxel in patients with human epidermal growth factor receptor 2-positive metastatic breast cancer administered as first-line treatment: the M77001 study group. J Clin Oncol. (2005) 23:4265-74. doi: 10.1200/JCO.2005.04.173

24. Olson EM, Abdel-Rasoul M, Maly J, Wu CS, Lin NU, Shapiro CL. Incidence and risk of central nervous system metastases as site of first recurrence in patients with HER2-positive breast cancer treated with adjuvant trastuzumab. Annals Oncol. (2013) 24:1526-33. doi: 10.1093/annonc/mdt036

25. Zhang Q, Chen J, Yu X, Cai G, Yang Z, Cao L, et al. Survival benefit of anti-HER2 therapy after whole-brain radiotherapy in HER2-positive breast cancer patients with brain metastasis. Breast Cancer. (2016) 23:732-9. doi: 10.1007/s12282-015-0631-x

26. Terrell-Hall TB, Nounou MI, El-Amrawy F, Griffith IGJ, Lockman PR. Trastuzumab distribution in an in vivo and in vitro model of brain metastases of breast cancer. Oncotarget. (2017) 8:83734-44. doi: 10.18632/oncotarget.19634

27. Mehta AI, Brufsky AM, Sampson JH. Therapeutic approaches for HER2positive brain metastases: circumventing the blood-brain barrier. Cancer Treatment Rev. (2013) 39:261-9. doi: 10.1016/j.ctrv.2012.05.006

28. Bachelot T, Romieu G, Campone M, Dieras V, Cropet C, Dalenc F, et al. Lapatinib plus capecitabine in patients with previously untreated brain metastases from HER2-positive metastatic breast cancer (LANDSCAPE): a single-group phase 2 study. The Lancet. Oncology. (2013) 14:64-71. doi: 10.1016/S1470-2045(12)70432-1

29. Freedman RA, Gelman RS, Anders CK, Melisko ME, Parsons HA, Cropp AM, et al. TBCRC 022: a phase II trial of neratinib and capecitabine for patients with human epidermal growth factor receptor 2-positive breast cancer and brain metastases. J Clin Oncol. (2019) 37:1081-9. doi: 10.1200/JCO.18.01511

30. Krop IE, Lin NU, Blackwell K, Guardino E, Huober J, Lu M, et al. Trastuzumab emtansine (T-DM1) versus lapatinib plus capecitabine in patients with HER2positive metastatic breast cancer and central nervous system metastases: a retrospective, exploratory analysis in EMILIA. Annals Oncol. (2015) 26:113-9. doi: 10.1093/annonc/mdu486

31. Jiang Z, Yan M, Hu X, Zhang Q, Ouyang Q, Feng J, et al. Pyrotinib combined with capecitabine in women with HER2 + metastatic breast cancer previously treated with trastuzumab and taxanes: a randomized phase III study. J Clin Oncol. (2019) 37:2610-9. doi: 10.1200/JCO.2019.37.15_suppl.1001

32. Ibrahim EM, Kazkaz GA, Al-Mansour MM, Al-Foheidi ME. The predictive and prognostic role of phosphatase phosphoinositol-3 (PI3) kinase (PIK3CA) mutation in HER2-positive breast cancer receiving HER2targeted therapy: a meta-analysis. Breast Cancer Res Treatment. (2015) 152:463-76. doi: 10.1007/s10549-015-3480-6

33. Wang Y, Liu Y, Du Y, Yin W, Lu J. The predictive role of phosphatase and tensin homolog (PTEN) loss, phosphoinositol-3 (PI3) kinase (PIK3CA) mutation, and PI3K pathway activation in sensitivity to trastuzumab in HER2positive breast cancer: a meta-analysis. Current Med Res Opinion. (2013) 29:633-42. doi: 10.1185/03007995.2013.794775

34. Swanton C. Intratumor heterogeneity: evolution through space and time. Cancer Res. (2012) 72:4875-82. doi: 10.1158/0008-5472.CAN-12-2217

35. Yap TA, Gerlinger M, Futreal PA, Pusztai L, Swanton C. Intratumor heterogeneity: seeing the wood for the trees. Sci Transl Med. (2012) 4:127ps10. doi: 10.1126/scitranslmed.3003854

36. Murtaza M, Dawson SJ, Tsui DW, Gale D, Forshew T, Piskorz AM, et al. Noninvasive analysis of acquired resistance to cancer therapy by sequencing of plasma DNA. Nature. (2013) 497:108-12. doi: 10.1038/nature12065

37. Berns K, Horlings HM, Hennessy BT, Madiredjo M, Hijmans EM, Beelen K, et al. A functional genetic approach identifies the PI3K pathway as a major determinant of trastuzumab resistance in breast cancer. Cancer Cell. (2007) 12:395-402. doi: 10.1016/j.ccr.2007.08.030

38. Saal LH, Holm K, Maurer M, Memeo L, Su T, Wang X, et al. PIK3CA mutations correlate with hormone receptors, node metastasis, and ERBB2, and are mutually exclusive with PTEN loss in human breast carcinoma. Cancer Res. (2005) 65:2554-9. doi: 10.1158/0008-5472-CAN-0 4-3913

39. Park SE, Park K, Lee E, Kim JY, Ahn JS, Im YH, et al. Clinical implication of tumor mutational burden in patients with HER2-positive refractory metastatic breast cancer. Oncoimmunology. (2018) 7:e1466768. doi: 10.1080/2162402X.2018.1466768

Conflict of Interest: The authors declare that the research was conducted in the absence of any commercial or financial relationships that could be construed as a potential conflict of interest.

Copyright (C) 2020 Chen, Ouyang, Anwar, Xie, Wang, Fan, Qian, Chen, Zhou, Guo, Gu, Ding, Yang, Liu, Deng, Xiao, Li, Wang, Zeng, Hu, Zhou, Qiu, Wang, Weng, Liu, $L i$, Tang, Wang, Zhang, Dai, Tang, Wu, Xiao, Li, Liu, Li, Yi and Ouyang. This is an open-access article distributed under the terms of the Creative Commons Attribution License (CC BY). The use, distribution or reproduction in other forums is permitted, provided the original author(s) and the copyright owner(s) are credited and that the original publication in this journal is cited, in accordance with accepted academic practice. No use, distribution or reproduction is permitted which does not comply with these terms. 